\title{
Simulation of Local and Global Atrophy in Alzheimer's Disease Studies
}

\author{
Oscar Camara-Rey ${ }^{1}$, Martin Schweiger ${ }^{1}$, Rachael I. Scahill ${ }^{2}$, \\ William R. Crum ${ }^{1}$, Julia A. Schnabel ${ }^{1}$, Derek L.G. Hill ${ }^{1}$, and Nick C. Fox ${ }^{2}$ \\ ${ }^{1}$ Center of Medical Image Computing, Unviersity College of London, UK \\ o.camara-rey@ucl.ac.uk \\ ${ }^{2}$ Dementia Research Centre, Institute of Neurology, \\ University College of London, $\mathrm{UK}^{\star}$
}

\begin{abstract}
We propose a method for atrophy simulation in structural MR images based on finite-element methods, providing data for objective evaluation of atrophy measurement techniques. The modelling of diffuse global and regional atrophy is based on volumetric measurements from patients with known disease and guided by clinical knowledge of the relative pathological involvement of regions. The consequent biomechanical readjustment of structures is modelled using conventional physics-based techniques based on tissue properties and simulating plausible deformations with finite-element methods. Tissue characterization is performed by means of the meshing of a labelled brain atlas, creating a reference volumetric mesh, and a partial volume tissue model is used to reduce the impact of the mesh discretization. An example of simulated data is shown and a visual evaluation protocol used by experts has been developed to assess the degree of realism of the simulated images. First results demonstrate the potential of the proposed methodology.
\end{abstract}

\section{Introduction}

The pattern of cerebral atrophy on magnetic resonance imaging (MRI) is currently used to help differentiate the degenerative dementias while the rate of atrophy may be used to detect early disease and potentially to assess diseasemodifying effects of therapies. Algorithms referred to as computational anatomy [1] are computerized approaches that offer automated or semi-automated solutions for MRI analysis, including quantification of cerebral atrophy. There is a lack of a gold standard against which to judge these methods or to help refine them. There have been limited attempts 234] to model the complex effects on

\footnotetext{
O O. Camara acknowledges support of the EPSRC GR/S48844/01, Modelling, Understanding and Predicting Structural Brain Change. W.R. Crum and M. Schweiger acknowledge support of the Medical Images and Signals IRC (EPSRC GR/N14248/01 and UK Medical Research Council Grant No. D2025/31). J.A. Schnabel acknowledges support of the EPSRC GR/S82503/01, Integrated Brain Image Modelling project. R.I. Scahill and N.C. Fox acknowledge support from the UK Medical Research Council, G90/86 and G116/143 respectively.
} 


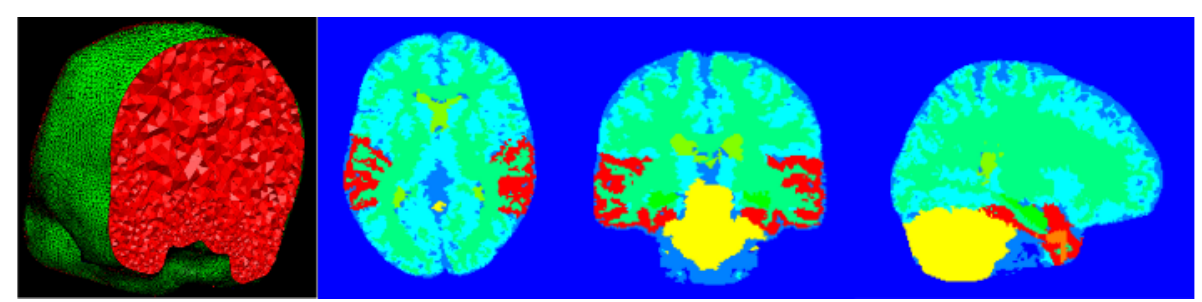

Fig. 1. Mesh generation procedure. Cutting plane of the volumetric finite-element mesh (left) and slices of the rasterization image generated from the labelled mesh, in which some of the used labels are shown (X11 colors): GM, light green; WM, cyan; cortical CSF, cornflowerblue; ventricles, greenyellow; MTL grey matter, red; subtentorial structures, yellow; hippocampi, limegreen; ERA, dark orange.

regional structural volumes and structural configuration that are a consequence of neurodegeneration. The main drawback of these approaches is that they do not take into account the interrelation of different tissue types.

We present a method for the simulation of atrophy in MR images of the brain in which atrophy is simulated in different tissue compartments and/or in different neuroanatomical structures with a phenomenological model. This model applies a differential pathological burden in different brain regions. Once volume changes have been defined in particular structures and tissues, the overall impact on brain shape is determined by a conventional physics-based biomechanical model. The consequent readjustment of structures is modelled using techniques based on tissue properties and simulating plausible deformations with finite-element methods (FEM). This validation framework was proposed by Schnabel et al. 5], for the analysis of MR mammography data. In our application, a thermoelastic model of tissue deformation is employed, controlling the rate of progression of atrophy by means of a set of thermal coefficients, each one corresponding to a different type of tissue.

The proposed technique can be divided into three main steps: meshing of a reference labelled brain atlas; its introduction into a FEM solver that will generate the simulated deformations; and the application of such deformations to the grey-level version of the brain atlas. A visual evaluation protocol used by experts has been developed to assess the degree of realism of the atrophy simulated images.

\section{Mesh Generation}

In this work, we have combined information from two well-known brain atlases to generate the reference labelled image and mesh used by the FEM solver: the Montreal Neurological Institute (MNI) Brainweb 11; and the International Consortium of Brain Mapping (ICBM) 2 atlases.

\footnotetext{
1 http://www.bic.mni.mcgill.ca/brainweb/

2 http://www.loni.ucla.edu/ICBM/
} 
For our purposes, we have taken into account the tissue labels from the MNI atlas, including the cerebrospinal fluid (CSF), the grey matter (GM) and the white matter (WM). We have also incorporated labels for the hippocampi and the lateral ventricle, obtained from a semi-automatic segmentation by clinical experts 6], due to their importance as biomarkers in dementia. In addition, we have incorporated the cerebral lobe labels from the ICBM Probabilistic atlas using an affine registration technique in order to place this atlas into the same geometrical space as the MNI one. Finally, two additional labels from the ICBM Template atlas have been included: a label covering the subtentorial structures to specify boundary conditions of the FEM; and a label for the entorhinal area (ERA) because of its implication in Alzheimer's disease. Some of these labels are illustrated in Figure 1

The first phase of the meshing consists of the generation of a surface mesh from the intracranial surface using the classical marching cubes [7] algorithm, followed by some post-processing steps (decimation, smoothing and selection of biggest connected component) to guarantee the creation of a continuous surface brain mesh. A volume (3D) finite-element (4-noded tetrahedra) mesh was then obtained from the intracranial mesh surface using the NETGEN 3 openSource software. We generated a tetrahedral mesh with very fine resolution (163765 nodes and 868404 elements, with 61132 surface elements). The final step is the labelling of the 3D mesh using the labelled voxel atlas, in which a set of particular biomechanical properties is assigned to each tetrahedron through a rasterization procedure [8]. In order to reduce the impact of the discretization of the mesh, we apply a partial volume tissue model to each tetrahedron in the FEM solver, as detailed in Section 3.3 .

\section{Finite-Element Deformation Model}

\subsection{Model}

The deformation model employs a linear elastic finite-element method based on the freely available TOAST package 4. In this work we considered structural displacements induced by atrophy that are small in relation to the total brain volume, so that the use of a linear model is justified.

The implementation of the FEM model follows a standard approach, whereby each tetrahedral mesh element is assigned a set of elastic material properties represented by an elasticity matrix D. In the case of isotropic elastic deformations, D is symmetric and can be expressed in terms of two parameters, usually given as Young's modulus $E$ and Poisson's ratio $\nu$. The elastic coefficients are assumed time-invariant. The deformation of the mesh is induced by assigning an isotropic thermal expansion coefficient $\alpha^{(i)}$ to each element $i$, and simulating a global temperature change $\Delta T$. The resulting isotropic thermal expansion enters the description of elastic deformation in the form of an initial element strain,

\footnotetext{
3 http://www.hpfem.jku.at/netgen/

4 http://www.medphys.ucl.ac.uk/\$ $\backslash \operatorname{sim} \$$ martins/toast/index.html
} 


$$
\varepsilon_{0}^{(i)}=\left\{\alpha^{(i)} \Delta T, \alpha^{(i)} \Delta T, \alpha^{(i)} \Delta T, 0,0,0\right\},
$$

where the relation between stresses $\sigma$ and strains $\varepsilon$ is given by

$$
\sigma^{(i)}=\mathrm{D}^{(i)}\left(\varepsilon^{(i)}-\varepsilon_{0}^{(i)}\right)+\sigma_{0}^{(i)}
$$

Assembling all element contributions of the mesh leads to the linear system

$$
\mathrm{Ku}+\mathbf{f}+\mathbf{f}^{\prime}=\mathbf{r}, \quad K_{i j}=\int_{V} \mathrm{~B}_{i}^{T} \mathrm{DB}_{j} d V
$$

with stiffness matrix $\mathrm{K}$. In an $n$-noded element, $\mathrm{B}$ is a $6 \times 3 n$ strain displacement matrix $\mathrm{B}=\left\{\mathrm{B}_{i}\right\}$, and

$$
\mathbf{f}^{\prime}=\int_{V} \mathrm{~B}^{T} \mathrm{D} \varepsilon_{0} d V,
$$

contains the volume forces arising from the initial thermal strain, $\mathbf{f}$ combines all other surface and volume force terms, $\mathbf{r}$ defines explicit displacements, and $\mathbf{u}$ is the vector of nodal displacements.

\subsection{Boundary Conditions and Mechanical Properties of Brain Tissue}

The surface of the mesh was assumed to coincide with the inner surface of the skull, and homogeneous Dirichlet boundary conditions were introduced using a Payne-Irons method to suppress the displacements of boundary nodes. The same strategy was applied to the mesh nodes corresponding to the subtentorial structures since atrophy induced by dementia is normally restricted to the supratentorial area.

There is no consensus on the optimal Young's modulus E and Poisson's ratio $\nu$ of the different brain tissues. Nevertheless, the choice of the elastic properties are not critical in the model presented here, because the boundary conditions enforce a constant global volume, while the relative changes in tissue region volumes are driven by the ratio of thermal coefficients. Furthermore, the mechanical properties of brain tissue are directly relevant to how the brain responds to an external force but are much less relevant to the response of the brain to diffuse cell damage and death that results in active structural readjustment. Therefore, we have adopted the shear modulus values proposed by McCracken et al. 9]: $12 \mathrm{KPa}$ for WM and 8KPa for GM (correspondingly, Young's modulus of 3.86KPa for $\mathrm{WM}$ and $2.57 \mathrm{KPa}$ for $\mathrm{GM}$ ). We consider the remaining structures, except the CSF, as being composed of an equal percentage of GM and WM, then their material properties are the average of the ones corresponding to the GM and WM $(E=3.22 \mathrm{KPa})$. For the Poisson's ratio, we have chosen a value of 0.45 for each brain tissue, except for the CSF, assumed to be a very soft and compressible tissue, with low Young's modulus $(E=0.1 \mathrm{KPa})$ and Poisson's ratio $(\nu=0.05)$.

\subsection{Thermal Coefficient Computation}

Given a segmentation of domain $\Omega$ into $T$ tissue types or anatomical structures $\Omega_{j}, \Omega=\cup_{j} \Omega_{j}, j=1 . . T$, we assume the thermal coefficient $\alpha$ to be constant 

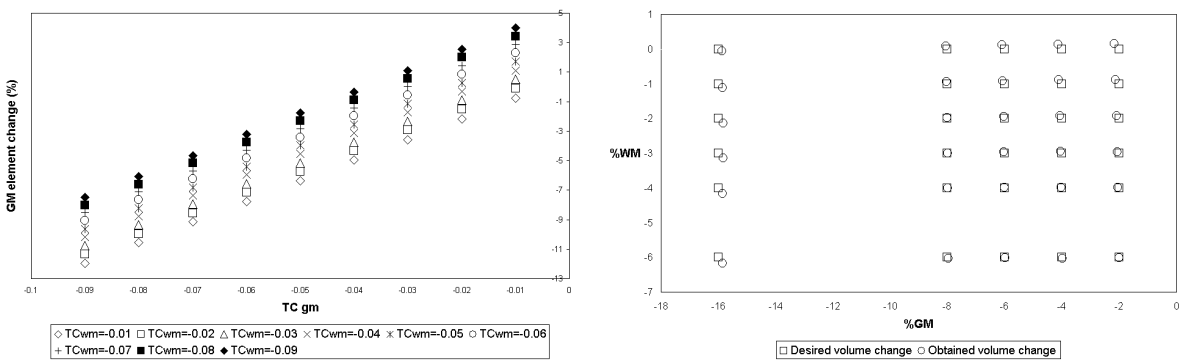

Fig. 2. Relation between thermal coefficients and desired volume change. Left: Percentage of GM element change when varying the thermal coefficients of the GM and WM (TCgm and TCwm respectively). Right: Relation between desired and the obtained volume change of GM and WM.

within each region $\Omega_{j}$, so that $\alpha$ is expressed in a piecewise constant basis, $\alpha(\mathbf{r})=\alpha_{j}, \mathbf{r} \in \Omega_{j}$. Once the material properties have been defined, we need to estimate the thermal coefficients $\alpha_{j}$ that will provide a mesh deformation consistent with the desired volume change $\Delta V_{j}$ in each tissue segment $\Omega_{j}$. This volume change will provide the ground-truth information needed for validation. $\Delta V_{j}$ is computed by integrating the differences in element volumes between the original and displaced meshes over each region $\Omega_{j}$.

The method of recovering the thermal coefficients for a given set of target region volume changes is based on a linear inversion technique. The relationship between changes of $\alpha_{j}$ and $\Delta V_{i}$ in regions $i$ and $j$ is expressed by the Jacobian matrix

$$
\mathrm{J}_{i j}=\left[\frac{\partial V_{i}}{\partial \alpha_{j}}\right],
$$

which we compute with a finite difference scheme, by explicitly perturbing each $\alpha_{j}$. Now we form the linear system $\mathrm{J}[\alpha]=[\Delta V]$, which, taking into account the boundary conditions, is quadratic of dimension $(T-1) \times(T-1)$ and can be solved by a standard LU decomposition. As illustrated in Figure 2, the assumption of linearity is appropriate. In this figure, we also show the good agreement between the desired and the obtained volume change for a set of 30 simulations (mean difference of $0.35 \pm 0.41$ and $0.14 \pm 0.08$ for GM and WM, respectively), confirming the approppriateness of the linear model.

As pointed out in Section 2, our framework enables us to use the information about the percentages of tissue in each element in order to reduce the effect of the mesh discretization through partial volume weighting. Hence, for each mesh element $i$, the thermal coefficient $\alpha^{(i)}$ will be a partial volume-weighted average of the region thermal coefficients contributing to the element:

$$
\alpha^{(i)}=\sum_{j} p_{j}^{(i)} \alpha_{j}
$$

where $p_{j}^{(i)}$ is the fractional volume contribution of region $j$ to element $i$. 


\section{Warping}

In order to simulate atrophy in the atlas image, we need to use interpolation techniques to generate a displacement field at each voxel of the image. We do this by weighting the displacement vectors of the nodes in the element containing a given voxel by the element linear shape function [10, and then the new image intensities can be interpolated using a truncated sinc interpolation kernel.

We have adopted a different interpolation strategy outside the mesh, where no deformation information is available from the FEM model. Here we employ a scattered data interpolation technique based on multilevel B-splines, as in Schnabel et al. 5 .

Using such an interpolation scheme, we can warp the grey-level version of the MNI atlas without noise using the dense displacement field generated by the FEM solver. Finally, we add Rician distributed noise to the atrophy simulated image.

\section{Simulation Example}

The illustrative example shown in this section is focused on Alzheimer's disease, as it is the most common cause of dementia. Therefore, the longitudinal example is based on the generation of a set of simulated images approximately mimicking the distribution of regional change in $\mathrm{AD}$ according to the severity of the disease. Three different atrophy simulated images are generated: in the first one $(t 1)$, only changes in the hippocampi and the entorhinal area are applied; the second image $(t 2)$ also includes GM changes in the medial temporal lobe and an expansion of the lateral ventricles; finally, a global reduction of the whole brain is applied to generate the third simulated image $(t 3)$. In addition, we have generated a corresponding set of simulated images at the three timepoints representing volume change induced by normal aging. We chose a range of values (see Table 1) which included a range of cerebral volume losses, as a percentage of baseline, that is consistent with published rates of atrophy (e.g. for hippocampus and brain) and study durations 1112 of 6-24 months Figure 3 shows the difference images between the normal aging and the AD simulated images at each timepoint.

\section{Measuring Degree of Realism}

The aim of this modelling is to generate simulated atrophy for the validation of longitudinal or cross-sectional techniques for the quantification of local and global atrophy. In order to determine whether our simulated atrophy was plausible, we designed an experiment in which a visual protocol is used by experts to differentiate between real and simulated MRI data of controls and patients with atrophy. The experiment uses two matched cohorts of 20 healthy elderly controls and 20 individuals with a diagnosis of probable Alzheimer's disease, together with nine atrophy simulated images. T1-weighted volumetric MR images were acquired on a 1.5 Tesla Signa unit (General Electric, Milwaukee). 
Table 1. Volume changes (\% with respect to the baseline) simulated in the longitudinal example. MTL: Medial Temporal Lobe. Most significant volume changes in the AD patients in each timepoint are marked in bold.

\begin{tabular}{|c||c|c|c||c|c|c||}
\hline \multirow{2}{*}{\multicolumn{1}{|c||}{ Structures }} & \multicolumn{3}{c||}{ Normal aging } & \multicolumn{3}{c||}{ Alzheimer's disease } \\
\cline { 2 - 7 } & $\mathrm{t} 1$ & $\mathrm{t} 2$ & $\mathrm{t} 3$ & $\mathrm{t} 1$ & $\mathrm{t} 2$ & $\mathrm{t} 3$ \\
\hline \hline Cortical CSF & 1.90 & 3.76 & 5.66 & 1.98 & 5.27 & $\mathbf{1 6 . 8 2}$ \\
\hline Ventricles & 2.18 & 4.31 & 6.49 & 2.23 & $\mathbf{5 . 8 2}$ & $\mathbf{1 9 . 3 4}$ \\
\hline Grey Matter & -0.65 & -1.28 & -1.92 & -0.65 & -1.30 & $\mathbf{- 5 . 3 1}$ \\
\hline White Matter & -0.17 & -0.32 & -0.49 & -0.16 & -0.32 & $\mathbf{- 1 . 3 3}$ \\
\hline Hippocampi & -1.40 & -2.79 & -4.21 & $\mathbf{- 3 . 3 3}$ & $-\mathbf{6 . 3 9}$ & $\mathbf{- 9 . 3 1}$ \\
\hline Subtentorial & 0.02 & 0.03 & 0.05 & 0.02 & 0.04 & 0.13 \\
\hline Entorhinal Area & -2.91 & -5.80 & -8.67 & $\mathbf{- 5 . 1 1}$ & $\mathbf{- 9 . 6 0}$ & $\mathbf{- 1 3 . 6 8}$ \\
\hline MTL Grey Matter & -0.63 & -1.28 & -1.92 & -0.65 & $\mathbf{- 4 . 6 8}$ & $\mathbf{- 8 . 5 9}$ \\
\hline Whole Brain & -0.36 & -0.71 & -1.08 & -0.38 & -1.01 & $\mathbf{- 3 . 2 0}$ \\
\hline
\end{tabular}
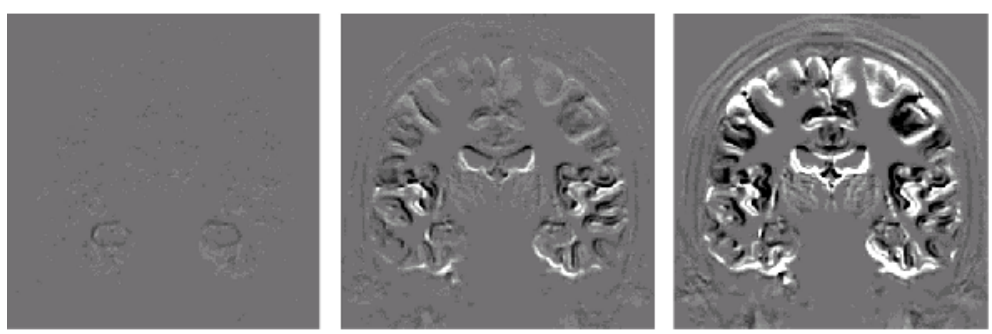

Fig. 3. Results of the longitudinal simulation example. Coronal slices of the difference images between the normal aging and the AD simulated images at each timepoint (from left to right, $t 1, t 2, t 3$ ).

A mesh warping [13] technique is employed to put the volumetric mesh presented in Section 2 into the anatomical space of nine of the controls, thus generating nine patient-specific meshes. Thereafter, they are introduced into the FEM solver, together with information about the desired volume change, which in this work we have obtained from volumetric difference estimates between both cohorts that have been previously computed using manual tracing techniques. The output of the FEM solver is a set of deformations that are applied to the control images to generate a group of atrophy simulated images equivalent to the cohort of mild AD subjects in terms of regional atrophy. The three sets of real and simulated data are then presented to the neurologists, who were blinded to scan information and were asked to classify each scan on a five point scale, with one being definite control, and five being definite AD.

The mean sensitivity and specificity for visual assessment of true AD and control scans was $90 \%$ and $77 \%$ respectively. The inter-rater agreement was moderate, with a kappa score of 0.52 . The mean score for each scan category is (mean $\pm \mathrm{STD}$ ): $1.95 \pm 1.42$ for controls; $4.23 \pm 3.19$ for real $\mathrm{AD} ; 3.19 \pm 1.36$ for simulated AD. The mean rating for the simulated atrophy scans was between that given for the control and the AD group. The simulated scans were not consistently classified as AD, but our results show that even true AD cases may 
be misidentified in one in ten cases. This may be due to the large inter-subject variation which exists when these subject groups are compared cross-sectionally. These results demonstrate that our simulation is sufficiently realistic to cause experienced neurologists to mis-classify some of our simulated images, and this supports the use of our simulated data in the validation algorithms that quantify atrophy.

\section{Conclusions}

We have proposed a method to generate valuable gold standard data for the testing and objective quantitative validation of atrophy measurement techniques. Our technique uses expert clinical knowledge of the rate and spatial distribution of atrophy in dementia to create a phenomenological model of the appearance of atrophy in MRI based on finite-element methods. The model includes structural remodelling concomitant with atrophy. Future work will focus on the generation and dissemination of several cohorts of data simulating the pattern of changes induced in the human brain by different types of dementia such as fronto-temporal dementia, vascular disease or multiple sclerosis. The simulated scans will be made available to the wider research community.

\section{References}

1. Ashburner, J., Csernansky, J., Davatzikos, C., Fox, N., et al.: Computer-assisted imaging to assess brain structure in healthy and diseased brains. Lancet Neurology 2 (2003) 79-88

2. Lao, Z., Shen, D., Xue, Z., Karacali, B., et al.: Morphological classification of brains via high-dimensional shape transformations and machine learning methods. Neuroimage 21 (2004) 46-57

3. Chen, K., Reiman, E., Alexander, G., Bandy, D., et al.: An automated algorithm for the computation of brain volume change from sequential MRIs using an iterative principal component analysis and its evaluation for the assessment of whole-brain atrophy rates in patients with probable Alzheimer's disease. Neuroimage 22 (2004) 134-143

4. Xue, Z., Shen, D., Davatzikos, C.: CLASSIC: Consistent Longitudinal Alignment and Segmentation for Serial Image Computing. Neuroimage 21 (2005) 46-57

5. Schnabel, J., Tanner, C., Castellano-Smith, A., Leach, M., et al.: Validation of Non-Rigid Registration using Finite Element Methods. In: Information Processing in Medical Imaging (IPMI'01). Volume 2082. (2001) 183-189

6. Freeborough, P., Fox, N., Kitney, R.: Interactive algorithms for the segmentation and quantitation of 3-D MRI brain scans. Computer Methods and Programs in Biomedicine 53 (1997) 15-25

7. Lorensen, W., Cline, H.: Marching cube, a high resolution 3D surface reconstruction algorithm. In: International Conference on Computer Graphics and Interactive Techniques (SIGGRAPH'87). Volume 21. (1987) 163-169

8. Sermesant, M., Forest, C., Pennec, X., Delingette, H., et al.: Deformable biomechanical models: Application to 4D cardiac image analysis. Medical Image Analysis 7 (2003) 475-488 
9. McCracken, P., Manduca, A., Felmlee, J., Ehman, R.: Mechanical Transient-Based Magnetic Resonance Elastography. Magnetic Resonance in Medicine 53 (2005) 628-639

10. Davies, A.J.: The Finite Element Method: A First Approach. Oxford University Press (1980)

11. Jack, Jr., C., Shiung, M., Gunter, J., O'Brien, P., et al.: Comparison of different MRI brain atrophy rate measures with clinical disease progression in AD. Neurology 62 (2004) 591-600

12. Schott, J., Price, S., Frost, C., Whitwell, J., et al.: Measuring atrophy in Alzheimer disease: A serial MRI study over 6 and 12 months. Neurology 52 (1999) 1687-1689

13. Camara, O., Crum, W., Schnabel, J., Lewis, E., Schweiger, M., Hill, D., Fox, N.: Assessing the quality of Mesh-Warping in normal and abnormal neuroanatomy. In: Medical Image Understanding and Analysis (MIUA'05). (2005) In Press. 